\title{
C-heterochromatin Variation in Deracantha onos (Pall.) (Deracanthini, Bradyporinae, Tettigoniidae, Orthoptera)
}

\author{
Elżbieta Warchałowska-Śliwa ${ }^{1}$ and Alexander G. Bugrov ${ }^{2}$ \\ ${ }^{1}$ Department of Experimental Zoology, Institute of Systematics and Evolution of Animals, \\ Polish Academy of Sciences, Slawkowska 17, 31-016 Karaków, Poland \\ ${ }^{2}$ Institute of Systematics and Ecology of the Siberian Branch, Russian Academy of \\ Sciences, Frunze 11, 630091 Novosibirsk and Department of Natural Sciences, \\ Novosibirsk State University, Pirogova 2, 630090 Novosibirsk, Russia
}

Accepted October 21, 1996

C-banding patterns have been demonstrated in a few species of Tettigoniidae (FernandezPiqueras et al. 1983, 1984, Navas-Castillo et al. 1986, Warchalowska-Śliwa and MaryańskaNadachowska 1992, 1995a, b, Warchalowska-Śliwa et al. 1993, 1994, 1995). Nevertheless, they have revealed that considerable intra- and inter-specific differences exist in the location and amount of constitutive heterochromatic regions.

So far, only the chromosome numbers of the tribe Deracanthini (sensu Čejchan 1967) of three species: Deracantha onos (=Callimenus onos) $2 \mathrm{n}=29, \mathrm{NF}=31$, Deracanthina deracanthoides, and Zychya baranovi $2 \mathrm{n}=31, \mathrm{NF}=31$ have been described (Ju 1930, Sergeev and Bugrov 1988).

The present work contains detailed information concerning the C-heterochromatin patterns in chromosomes of Deracantha onos.

\section{Material and methods}

Six males of Deracantha onos (Pallas 1772) were collected from two localities in Russia; 1) Transbaikalia, Chita region, near the village Olovyannaya and 2) Far East Siberia, Amur region, near Blagoveschensk (July 1982).

Four out of six males were injected with $0.1 \%$ colchicine for $1.5 \mathrm{hr}$. The testes were excised, incubated in $0.9 \%$ sodium citrate, fixed in ethanol-acetic acid $(3: 1)$, and kept in $70 \%$ ethanol. Air-dried preparations were made by squashing tissues in $45 \%$ acetic acid and freezing them in dry ice. The C-banding examination was carried out according to Sumner (1972) with slight modifications.

\section{Results}

The karyotype of Deracantha onos consists of $2 n=29$ (XO) chromosomes. The autosomes can be divided into three size groups; one long $\left(L_{1}\right)$, six medium $\left(M_{2}-M_{7}\right)$, and seven short $\left(S_{8}-\right.$ $\mathrm{S}_{14}$ ). Among fourteen autosomes, pair $\mathrm{L}_{1}$ is metacentric, while the others are acrocentric. The size of the acrocentric $\mathrm{X}$ chromosome is intermediate between that of the $\mathrm{L}$ and $\mathrm{M}$ autosomes (Fig. 1).

At diplotene/diakinesis in $\mathrm{L}_{1}$ bivalent from 4 to 6 chiasmata, in $\mathbf{M}_{2}$ two or sporadically one, in the remaining medium size one or sporadically two chiasmata, and in small bivalents only one chiasma was observed. The mean frequency of chiasmata is $\bar{x}=19.66( \pm 0.7)$.

This species is characterized by the occurrence of a large amount of C-band positive chromatin visible in early prophase (Fig. 2); they can be classified according to their location 


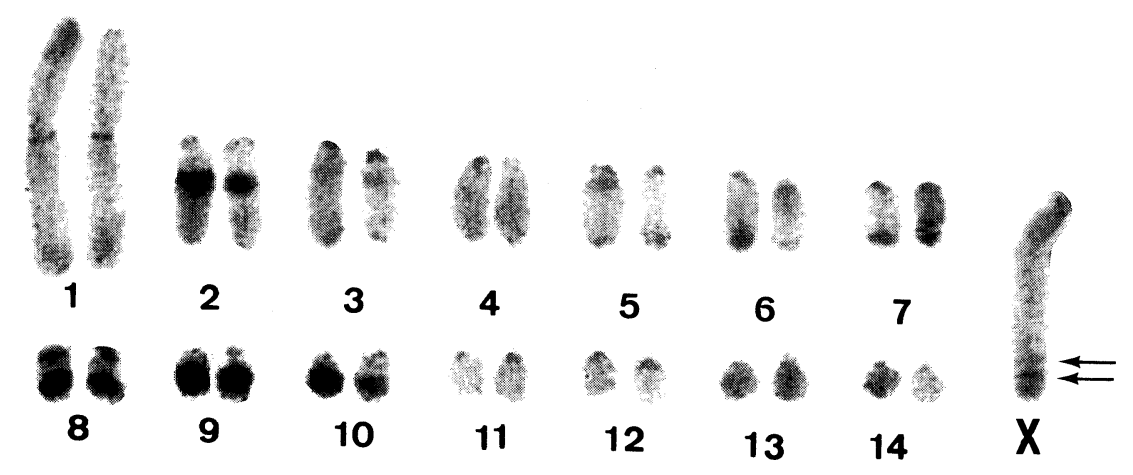

Fig. 1. C-banded karyotype. The $\mathrm{X}$ chromosome with interstitial C-bands (arows). Bar equals $10 \mu \mathrm{m}$.

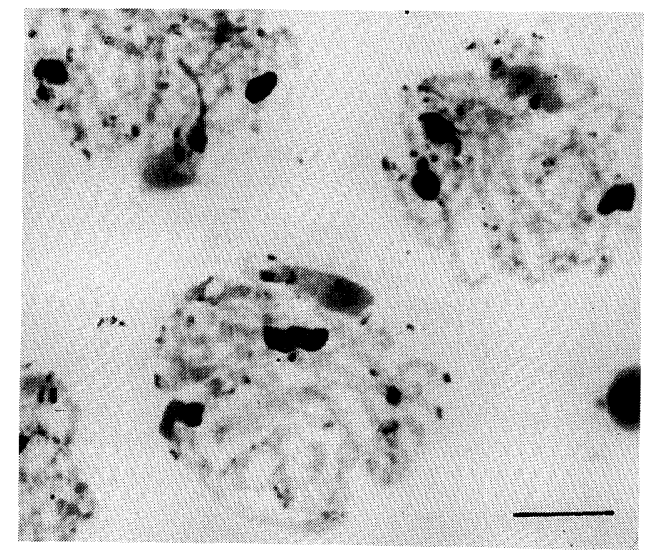

Fig. 2. Early prophase with positive chromatin. Bar equals $10 \mu \mathrm{m}$. within the chromosomes as follows: paracentromeric, interstitial, and distal.

The C-banding pattern in bushcrickets from two localities is characterized by the presence of paracentromeric C-bands in all autosomes and in the $\mathrm{X}$ chromosome (Fig. 1).

The interstitial C-bands are present in $\mathbf{M}_{2}$ chromosomes in individuals of both localities. C-heterochromatin variation for these autosomes is shown in Fig. 3a-f. In most of the individuals (found in three out of six individuals) $\mathbf{M}_{2}$ chromosomes carry isomorphic C-blocks (I-variant) (Fig. 3a,b). The II- and III-variants possesses heteromorphic C-bands of different size. Two males carry the II-variant of the $\mathbf{M}_{2}$, where one of the homologues shows a larger block than the other (Fig. $3 \mathrm{c}, \mathrm{d}$ ). The III-variant (found in one male) carries in one of the homologues a particularly large band (Fig. 3e,f). In diakinesis $\backslash \mathbf{M I}$ the $\mathbf{M}_{2}$ heteromorphic bivalent possesses two chiasmata in proximal + distal or interstitial + interstitial position, or one chiasma in a distal one (Fig. 4a). At anaphase I of this bivalent more often separated reductionally, than equitionally (Fig. 4b, c) owing to the formation of either one or two chiasmata.

Additionally, two thin interstitial C-bands were observed in all individuals in the $\mathrm{X}$ chromosome (Fig. 1).

Distal thin C-heterochromatin in the $\mathbf{M}_{3}, \mathbf{M}_{5}$, and $\mathbf{M}_{7}$ chromosomes was observed. The $\mathrm{S}_{8}$ and $\mathrm{S}_{9}$ chromosomes carry large distal C-blocks, whereas a small euchromatic part at the end of these chromosomes is present.

One male was mosaic for the heteromorphic distal C-band in $\mathbf{M}_{6}$ chromosomes (Figs. 1, 4d).

In all males the heteromorphic $S_{10}$ show two variants of distal C-bands; I-one of the homologues carring a larger block than the other (Figs. 1, 5a), II-one of homologues possesses a large block, whereas in the other one a small C-band was observed (Fig. 5b).

The two or three Ag-stained areas of the NORs continued in the meiotic prophase up to the pachytene stage (Fig. 6a). In diplotene/diakinesis the $M_{2}$ and one small size bivalents 


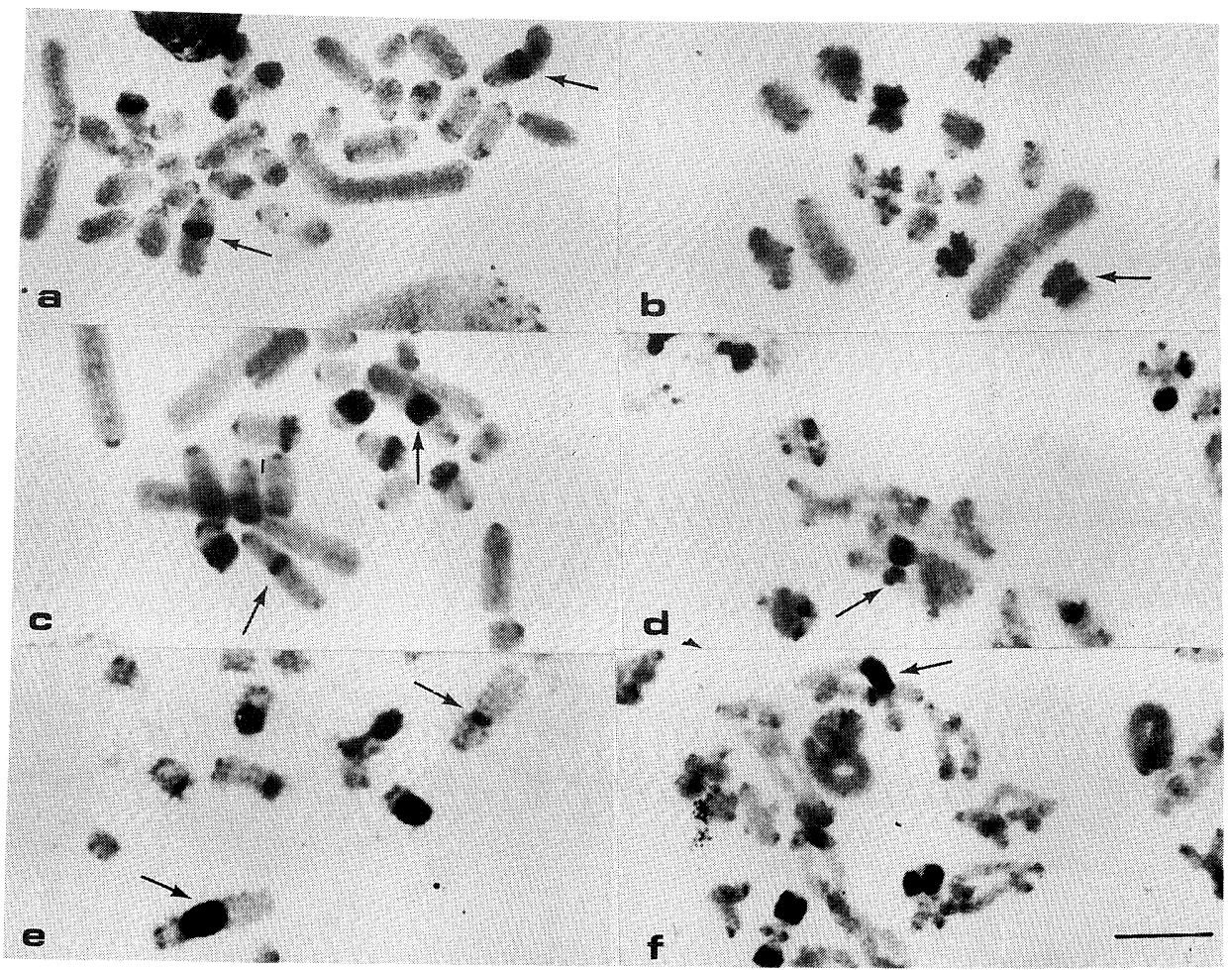

Fig. 3a-f. C-heterochromatin variation for $\mathbf{M}_{2}$ chromosomes (arrows). (a) and (b) I-variant in (a) mitotic metaphase and (b) metaphase I. (c) and (d) II-variant in (c) mitotic metaphase and (d) diplotene. (e) and (f) III-variant in (e) mitotic metaphase and (f) diplotene. Bar equals $10 \mu \mathrm{m}$.

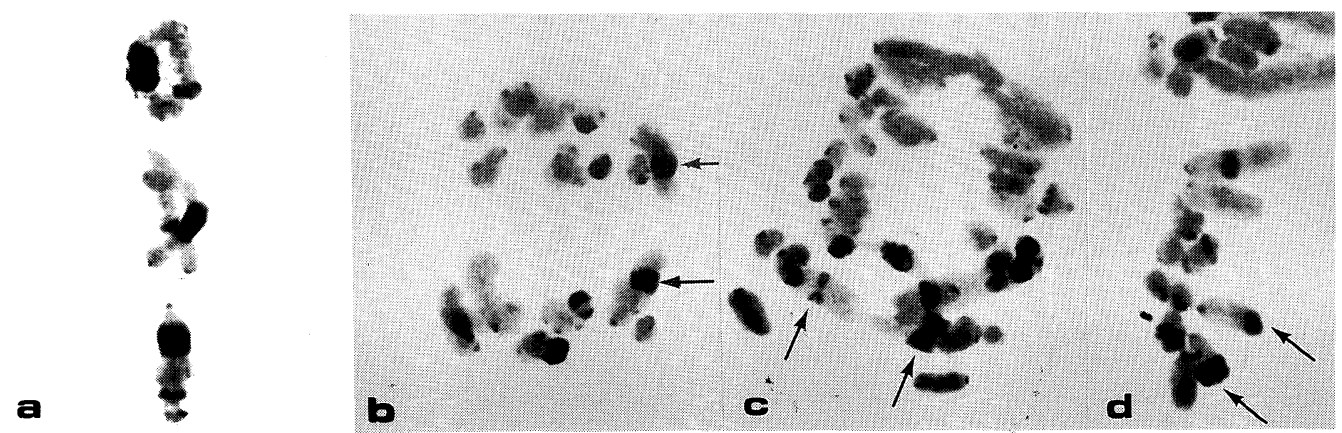

Fig. 4a-d. (a) $\mathrm{M}_{2}$ heteromorphic bivalent with different position of chiasmata. (b) Anaphase $I$, note in equitional and (c) reductional division of heteromorphic $\mathbf{M}_{2}$ bivalent (arrows). (d) Heteromorphic distal C-bands in $\mathbf{M}_{6}$ chromosomes (arrows). Bar equals $10 \mu \mathrm{m}$.

preserved nucleolar remnants. Sometimes, in the $\mathrm{X}$ chromosome small NOR is visible, and in most of the cells the $\mathrm{X}$ possesses many little dots (Fig. 6b).

\section{Discussion}

One of the most frequent categories of chromosome differences in Orthoptera involve change in the amount and locality of constitutive heterochromatin. This is evident in terms of the variation in the C-banded material. Particular C-bands are present or absent, and show 


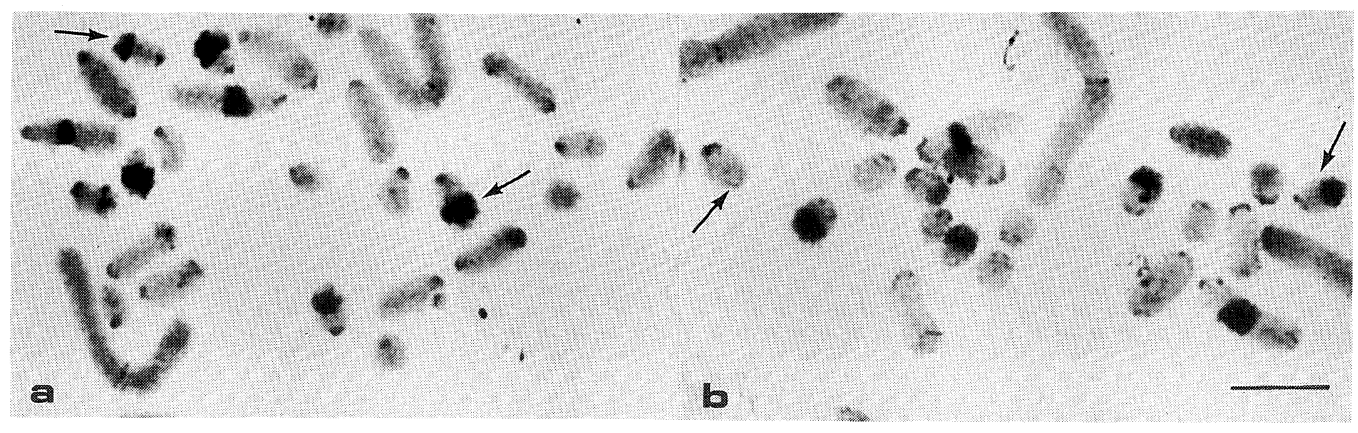

Fig. 5a, b. Heteromorphic distal C-bands in $\mathrm{S}_{10}$ chromosomes (arrows) (a) I-variant and (b) IIvariant. Bar equals $10 \mu \mathrm{m}$.

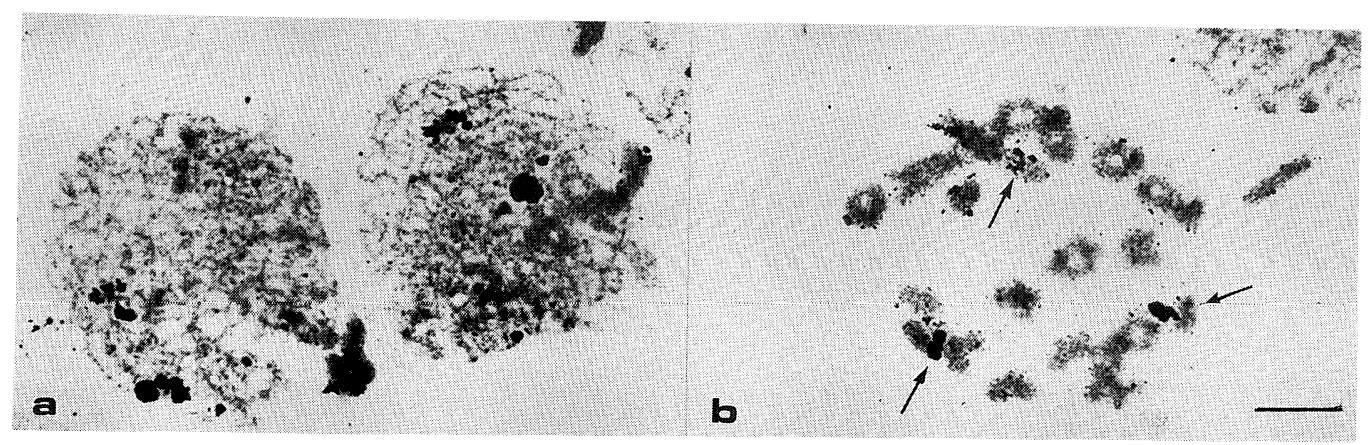

Fig. 6a,b. Silver stained (a) pachytene and (b) diakinesis showing the location of the NORs (arrows). Bar equals $10 \mu \mathrm{m}$.

variation in size. In most cases in Orthoptera variation of the C-heterochromatin in both homologous chromosome involve the shorter member of complements (Hewitt 1979, NavasCastillo et al. 1986).

Hitherto, variation for the heterochromatin C-bands in Bradyporinae has been found in the genus Beatica (Fernandez-Piqueras et al. 1984, Navas-Castillo et al. 1986, Sentis et al. 1986), and in the Steropleurus martorelli complex (Fernandez-Piqueras et al. 1983).

The present study analysed individuals of Deracantha onos from two localities which are very far and isolated from one another. Males in both localities showed three types of $\mathbf{M}_{2}$, two types of $\mathbf{M}_{6}$, and $\mathrm{S}_{10}$ chromosomes depending on the C-banding pattern. The C-heterochromatin variation in the common type is related to the species Baetica ustulata endemic in the Iberian Peninsula. This species, belonging to the tribe Ephippigerini, consists of $2 n=25$ chromosomes, where both pairs of $\mathrm{L}$ autosomes are metacentric while the remainder, including the $\mathrm{X}$, are acrocentric. In $\mathrm{L}_{2}$ chromosomes there are interstitial $\mathrm{C}$-bands in the long arms, which coincide with active NOR. Additionally, in this species extensive variation has been described in heterochromatin existing for the C-blocks in $\mathbf{M}_{3}, \mathbf{M}_{4}$, and $\mathbf{M}_{5}$ chromosomes (Fernandez-Piqueras et al. 1984, Navas-Castillo et al. 1986, Sentis et al. 1986). Between Baetica ustulata and Deracantha onos there are some similaries and differences within the location and size of C-heterochromatin: 1) These species are characterized by different chromosome number and by their morphology. One the one hand, there are the same numbers of small size autosomes, and on the other, differences between the number of large and medium chromosomes occurred. The supposed origin of metacentric $\mathbf{L}_{2}$ chromosomes was connected with mutual translocation of the largest of medium size chromosomes. 2) The analysis of 
C-banding patterns of different species of Tettigonidae, and the above-mentioned species reveals that the largest and medium size chromosomes possess small in size paracentromeric C-bands. 3) The $\mathbf{M}_{2}$ chromosomes in $D$. onos with heterochromatin variation in interstitial position and connected with it active NOR, could correspond with the long arm with interstitial C-band of $\mathbf{L}_{2}$ in $B$. ustulata. 4) In both species, with similar medium size autosomes, distal C-bands were present. However, the $\mathbf{M}_{6}$ chromosomes in $D$. onos was characterized by heteromorphic thin distal C-bands, but $\mathrm{M}_{5}$ in $B$. ustulata possesses some types of variation in the size of C-heterochromatin. 5) Unlike B. ustulata, D. onos posseses more C-heterochromatin in small size chromosomes, especially in $S_{8}, S_{9}$ and $S_{10}$. Thus differences in size and location of $\mathrm{C}$-heterochromatin in the two species generally occur in small size chromosomes. It could be said that the distribution and amount of C-heterochromatin in these species show a parallel tendency. Among all species of Tettigoniidae described so far in terms of C-band, distribution, amount, as well as variation of $\mathrm{C}$-heterochromatin are the most similar in the above-mentioned species. Hence, the cytogenetical data presented here, confirm in a certain way the uniting of the tribes Deracantini and Ephippigerini in one higher taxon, by some systematists (Čejchan 1967, Gorochov 1988).

\section{Summary}

The Mongolian-Siberian species Deracantha onos $(2 n=29, N F=31)$ is characterized by an extensive amount of C-heterochromatin occurring on the $\mathbf{M}_{2}$ chromosomes as a large interstitial C-block and on $\mathbf{M}_{6}, \mathrm{~S}_{8}, \mathrm{~S}_{9}$, and $\mathrm{S}_{10}$ chromosomes as large distal C-bands.

\section{References}

Čejchan, A. 1967. A taxonomic study in Deracanthinae (Orthoptera, Bradyporidae). Acta entomologica Musei Nationalis Pragae 37: 607-633.

Fernandez-Piqueras, J., Rodriquez-Campos, A. and Sentis-Castano, C. 1983. Hypotheses about speciation by chromosomal rearrangements in the Steropleurus martorelli complex (Tettigonioidea, Orthoptera). Genetica 60: $167-172$.

- , - - and Rojo-Garcia, E. 1984. C-heterochromatin in the monospecific genus Baetica (Orthoptera: Tettigoniidae). Caryologia 37: 69-76.

Gorochov, A. V. 1988. 4. The classification and phylogeny of Grasshoppers (Gryllida-Orthoptera, Tettigoniidae).Ed. A Ponomarenko. In: The Cretaceous Biocoenotic Crisis and the Evolution of Insects. Nauka, Moscow, pp. 145-190. (In Russian)

Hewitt, G. M. 1979. Grasshoppers and crickets. Animal Cytogenetics. Vol. 3: Insecta 1 Orthoptera. Ed. Gebruder Borntraeger. Berlin, Stuttgar.

Ju, C. L. 1930. Spermatogenesis and chromosomes of Callimenos onos (Pallas) (Tettigoniidae, Orthoptera). Peking Nat. Hist. Bull. 5: 1-24.

Navas-Castillo, J., Cabrerro, J. and Camacho, J. P. M. 1986. Heterochromatin variation in Baetica ustulata (Orthoptera: Tettigoniidae) analysed by $\mathrm{C}$ and $\mathrm{G}$ banding. Heredity 56: 161-165.

Sentis, C., Santos, J. and Fernandez-Piqueraqs, J. 1986. C-heterochromatin polymorphism in Baetica ustulata: intraindividual variation and fluorescence banding patterns. Chromosoma 94: 65-70.

Sergeev, M. G. and Bugrov, A. G. 1988. Katydids of subfamily Deracanthinae (Orthoptera, Bradyporidae) from Siberia. Taksonomiya Zhivotnych Sibiri, Russian Academy of Sciences, Novosibirsk: 46-53. (In Russian).

Sumner, A. T. 1972. A simple technique for demonstrating centromere heterochromatin. Exp. Cell. Res. 75: $304-306$.

Warchalowska-Śliwa, E. and Maryańska-Nadachowska, A. 1992. Karyotypes, C-bands, NORs location in spermatogenesis of Isophya brevipennis Brunner (Orthoptera: Phaneropteridae). Caryologia 45: 83-89.

- 1995a. Cytogenetic studies of the genus Tettigonia (Orthoptera, Tettigonioidea, Tettigoninae). I. C-bands and NORs activity. Folia biol. (Kraków) 43: 29-34.

- 1995b. Cytogenetic studies of the genus Tettigonia (Orthoptera, Tettigonioidea, Tettigoninae). II. Heteromorphism of C-bands. Folia biol. (Kraków) 43: 99-106.

— and Bugrov, A. G. 1993. Karyotypes, C-heterochromatin, and NORs in three species of the genus Gampsocleis Fieb. 
(Orthoptera: Tettigonioidea: Decticinae). Folia biol. (Kraków) 40: 119-127.

- - - and Maryańska-Nadachowska, A. 1994. Karyotypes, C-banding pattern, and NORs of the genus Montana Zeuner 1941 (Orthoptera, Tettigonioidea, Decticinae). Folia biol. (Kraków) 42: 89-94.

-, - and - 1995. Karyotypes of three species of the genera Poecilimon Fisch. and Isophya Br.-W. (Orthoptera, Tettigonioidea, Phaneropterinae) from the North Caucasus. Caryologia 48: 27-34. 